\title{
Química na Internet
}

\author{
JOÃO AIRES DE SOUSA*
}

\begin{abstract}
A Internet é apresentada resumidamente, em termos das suas origens, dimensão actual e principais serviços. São detalhadas as mais importantes aplicações que tem tido na Química e referidos alguns sítios virtuais particularmente úteis ou representativos.
\end{abstract}

\section{A INTERNET}

A Internet nasceu dum programa militar norte-americano dos anos 60, típico da Guerra Fria. O Departamento de Defesa pretendia desenvolver uma rede capaz de garantir linhas de comando e controlo numa situação pós - ataque nuclear. Foi então concebido um sistema que não tivesse nenhuma autoridade central e que funcionasse mesmo que feito em pedaços. Chamava-se ARPAnet a primeira rede que ligou, em 1969, 4 computadores (poderosíssimos para o tempo) e que era utilizada para partilhar os recursos computacionais entre 4 localidades universitárias. Isto abria possibilidades preciosas de programação à distância e... de troca de informação. Logo ao fim de dois anos, o sistema, que fundamentalmente se destinava a programação remota, estava transformado num verdadeiro serviço postal e a comunidade científica usava-o sobretudo em colaborações científicas, mas também para troca de mensagens pessoais ou simplesmente para falar da vida alheia! [1,2]

$\mathrm{O}$ crescimento da Internet (como passou a ser conhecida a partir dos anos 80) foi desde logo exponencial, acolhendo redes autónomas e computadores com configurações muito diferentes. Foi aberta ao público em 1987 [3] e existe ligação a Portugal desde 1991 [4]. Estima-se que a dimensão da rede duplique em cada ano e que, em Outubro de 1995, existiriam 7.7 milhões de computadores com ligação permanente [5], $26.4 \mathrm{mi-}$ lhões de utilizadores com acesso a serviços interactivos da Internet [5] e 39 milhões de utilizadores com acesso a correio electrónico [5], espalhados, pelo menos, por 168 países [6].
Mas o que é afinal a Internet? É uma rede de computadores (ou melhor, uma rede não hierarquizada de redes de computadores) que comunicam entre si através de protocolos estandardizados (TCP/IP). A utilização destes protocolos permite ligar computadores independentemente da sua arquitectura ou sistema operativo. A partir de um computador pode aceder-se a outro qualquer e operálo à distância, ou trocar ficheiros e software. Complementarmente, a Internet tem sido definida como o conjunto da informação contida nesta rede e das relações estabelecidas a partir dela [7].

Com estas potencialidades têm sido inventados serviços engenhosos para os quais foi desenvolvido software de muito fácil manuseamento e que torna o uso da Internet infinitamente mais simples do que pode fazer intuir qualquer apresentação no papel!

De seguida descrevemos resumidamente os principais serviços da Internet (E-mail, Usenet, FTP, Archie, Telnet e World Wide Web) e finalmente apresentamos as mais relevantes aplicações que estes têm encontrado na Química. Na Tabela I coleccionámos os endereços dos sítios virtuais referidos neste texto e de outros particularmente úteis ou representativos. É no entanto de salientar que a informação aqui apresentada estará rapidamente desactualizada uma vez que, por um lado, a localização dos documentos muda com grande frequência e, por outro, estão constantemente a aparecer funcionalidades novas na tecnologia Internet.

E-mail (ou correio electrónico): Permite a troca de mensagens ou ficheiros entre quaisquer dois utilizadores que possuam áreas de correio electrónico em computadores servidores (servers). O e-mail tem várias vantagens relativamente ao correio tradicional (ou ao fax): é instantâneo, é mais económico, pode ser lido pelo destinatário a partir de qualquer ponto da Intenet e permite enviar texto "vivo" que pode ser arquivado e manuseado por qualquer processador de texto ou base de dados. Tem a desvantagem de não ser possível garantir a confidencialidade e pode argumentar-se que não é tão pessoal...

Usenet (ou "News"): É um conjunto de fóruns de discussão, cada qual destinado ao seu tema, para onde qualquer utilizador que tenha uma área de correio electrónico pode enviar um contributo e cujas contribuições podem ser lidas por qualquer utilizador. Existem vários milhares de grupos de discussão que abrangem virtualmente todos os assuntos desde política ou religiões até chocolates ou música filipina incluindo, obviamente, Química. Alguns destes grupos são moderados, isto é, há um responsável pelo grupo que só torna públicos os contributos que julgue pertinentes. Para além das "News" existem "Mailing Lists" que funcionam de modo análogo mas com a diferença de que os participantes estão registados numa lista e as contribuições são-lhes enviadas por correio electrónico.

FTP (File Transfer Protocol): Permite a transferência de ficheiros entre dois computadores. Qualquer servidor de FTP pode restringir o acesso apenas aos utilizadores que tenham uma área e possuam um código (password) ou pode, por segurança, permitir exportar ficheiros mas não importar.

Archie: É um serviço de pesquisa de ficheiros disponíveis por FTP. Um computador que seja servidor de Archie tem uma listagem (periodicamente actualizada) dos ficheiros acessíveis por FTP numa determinada região da Internet. Utilizando o software adequado pode aceder-se a qualquer destes servidores de Archie e procurar na sua listagem os ficheiros que contenham uma determinada palavra. Uma vez localizados podem ser importados por FTP. É sobretudo útil para encontrar software.

Telnet: Com este serviço é possível a um utilizador aceder a um computador localizado noutro ponto da Internet e operá-lo, fazendo-o correr apli- 
cações ou manipulando ficheiros nele contidos. Também neste caso, o acesso pode ser público ou estar restringido a alguns computadores e/ou conhecedores duma password. Tem permitido a utilização de hardware muito poderoso por um grande número de utilizadores fisicamente distantes ou efectuar pesquisas em bases de dados muito vastas que, existindo num só local, estão assim disponíveis a partir de qualquer ponto.

World Wide Web (WWW ou Web): A WWW é o mais completo serviço da Internet e o mais fácil de utilizar. Assenta na ideia de hipermédia. Um documento hipermédia é um documento que integra texto, imagens (eventualmente som ou vídeo) e que contém ligações a outros documentos: algumas partes do documento estão destacadas (sublinhadas, de cor diferente ou contornadas, por exemplo) para indicar que basta clicar com o rato sobre elas para saltar para outra parte do mesmo documento ou para abrir outro ficheiro. Este novo ficheiro pode ser outro documento hipermédia ou um ficheiro qualquer presente num computador servidor e identificado por uma URL (Uniform Resource Locator). Por exemplo, se introduzir num programa para WWW a URL http://www.dq.fct.unl.pt/textos/quiminet.html ele importará e abrirá a versão WWW do artigo que está a ler e que se encontra num servidor da Faculdade de Ciências e Tecnologia no Monte da Caparica. Verificará que algumas palavras estão sublinhadas e aparecem com cor diferente. É o caso da expressão "World Wide Web" no início deste parágrafo: ao clicar sobre ela, será importado e aberto um documento que se encontra no CERN (Laboratório Europeu de Física das Partículas, Suiça) e que tem informação sobre a World Wide Web.

Num universo de milhões de documentos, como é o caso da Web, pode ser difícil encontrar a informação pretendida. Para ajudar nessa tarefa existem listas e índices globais (ver Tabela I) que são ainda rudimentares, mas muito úteis, onde é possível fazer pesquisas por palavraschave.

Para utilizar cada um destes serviços é preciso ter o software respectivo. Existem vários programas de distribuição livre para cada um e para as várias plataformas de computadores (Macintosh, Windows, Unix,...). Para ter acesso à Internet pode fazer-se uma ligação permanente (como acontece normalmente nas Universidades) ou temporária através duma vulgar linha telefónica com um modem. Há já pelo menos quatro empresas em Portugal que permitem esta última modalidade por uma mensalidade entre 2500\$00 e $5000 \$ 00$.

\section{APLICAÇÕES EM QUÍMICA}

\section{Um novo modo de comunicar Química}

A Internet tem sido apresentada como uma das mais importantes ferramentas científicas deste final de século $[1,8]$. Seguramente, o sucesso da investigação científica assenta cada vez mais na troca e disponibilidade de informação e a Internet fá-lo como jamais outro instrumento foi capaz. Milhões de documentos podem agora passar a estar disponíveis com o simples clicar do rato; os próximos anos vão com certeza multiplicá-los permanentemente e, sobretudo, organizá-los em sofisticados índices e bases de dados que permitirão realizar novos tipos de pesquisas e garantir a qualidade da informação encontrada.

Mas para além desta abundância extraordinária de informação, a Internet veio generalizar uma substancial alteração qualitativa [9]. A Química tem sido desde sempre comunicada a duas dimensões, seja no suporte do papel, do quadro, do diapositivo ou da transparência. O que não deixa de ser curioso, uma vez que as moléculas são essencialmente estruturas tridimensionais. Para ob- viar às limitações dos suportes bidimensionais inventaram-se soluções como as projecções de Fischer ou as projecções de Haworth, que são manifestamente insuficientes quando se trata de estruturas com a complexidade das proteínas ou quando se inspeccionam subtilezas mecanísticas de reacções estereoselectivas. Ora a Internet permite transferir estruturas moleculares em termos das suas coordenadas espaciais de forma a que, com um programa visualizador, possam ser rodadas e observadas a partir de um qualquer ângulo. Ou directamente utilizadas para iniciar uma sessão de modelação molecular, ou para utilização numa base de dados. A WWW torna tudo isto muito simples. Por exemplo, a Figura 1 mostra o documento de WWW com a URL http://www.ch.ic.ac.uk/ectoc/papers/50/ e que é um poster electrónico [10] apresentado na ECTOC-1 (Electronic Conference On Trends in Organic Chemistry). Basta clicar com o rato sobre a estrutura molecular nele contida para que sejam importadas as suas coordenadas moleculares e para que ela apareça no monitor; movimentando então o rato ela rodará perante os nossos olhos podendo ser inspeccionada a partir da posição mais conveniente!

Num "artigo electrónico" é ainda possível incluir ficheiros de som, sequências de vídeo, formulários para pesquisa imediata em bases de dados ou para efectuar cálculos num computador distante, ligações a referências bibliográficas ou até aos ficheiros com informação espectroscópica primária localizados no espectrómetro onde foram adquiridos!

Fica então evidente como é o próprio conceito de "publicação" que está em causa [11]. Qualquer investigador pode agora disponibilizar informação a toda a comunidade científica, em formatos totalmente novos, a partir do computador do seu laboratório ou gabinete, sem interferência de nenhuma "autoridade" ... Mas essa informação só será verdadeiramente pública se estiver referenciada em algum local aonde 
Tabela I - Selecção de locais de Química na Internet particularmente úteis ou representativos

\begin{tabular}{|c|c|c|}
\hline Nome & URL & Observações \\
\hline \multicolumn{3}{|c|}{ Departamentos de Química de Universidades Portuguesas } \\
\hline - Universidade do Minho & \multicolumn{2}{|l|}{ http://www-dq.ci.uminho.pt/depquimica.html } \\
\hline - Universidade do Porto & \multicolumn{2}{|l|}{ http://garfield.fe.up.pt:8001/portugues/qui/qui.html } \\
\hline - Universidade de Coimbra & \multicolumn{2}{|l|}{ http://www.eq.uc.pt/ } \\
\hline & \multicolumn{2}{|l|}{ http://cygnus.ci.uc.pt:80/bioquimica/ } \\
\hline • Universidade de Lisboa & \multicolumn{2}{|l|}{ http://www.fc.ul.pt/departs/quimica/index.html } \\
\hline - Instituto Superior Técnico & \multicolumn{2}{|l|}{ http://www.ist.utl.pt/ist/guia/estru.html } \\
\hline - Universidade Nova de Lisboa & \multicolumn{2}{|l|}{ http://www.dq.fct.unl.pt } \\
\hline - Universidade de Évora & \multicolumn{2}{|l|}{ http:/www.uevora.pt/Areas/Quimica/quimica.html } \\
\hline \multicolumn{3}{|c|}{ Listas de Recursos de Química } \\
\hline - Best of Chemistry '95 & http://www.ch.ic.ac.uk/infobahn/boc.html & Selecção criteriosa. \\
\hline - ChemDex Index, Sheffield & http://www.shef.ac.uk/ chem/chemistry-www-sites.html & Provavelmente a mais completa e organizada lista global de Química. \\
\hline \multicolumn{3}{|c|}{ Bases de Dados } \\
\hline - BIDS & $\begin{array}{l}\text { telnet://bids.ac.uk } \\
\text { (depois de ligado, escolher "Uncover Service") }\end{array}$ & $\begin{array}{l}\text { Pesquisa bibliográfica (cobre mais de } 15000 \text { títulos de } \\
\text { publicações). }\end{array}$ \\
\hline - Chemical Abstracts* & http://info.cas.org/welcome.html & Chemical Abstracts on-line. \\
\hline - Beilstein* & http://www.beilstein.com/ & Bases de dados Beilstein. \\
\hline - Hazardous Chem. Database & http://odin.chemistry.uakron.edu/erd/ & Na Universidade de Akron. \\
\hline - Material Safety Data Sheets & gopher://atlas.chem.utah.edu/11/MSDS & A colecção MSDS. \\
\hline \multicolumn{3}{|c|}{ Material de Ensino } \\
\hline - Chem. Teaching Resources & http://www.anachem.umu.se/eks/pointers.htm & Lista excepcional (Un.Umea,Suécia) \\
\hline - Global Instruct. Chemistry & http://www.ch.ic.ac.uk/GIC/ & Lista organizada por Henry Rzepa. \\
\hline - Schools Page & http://www.ch.ic.ac.uk/schools/ & Recursos para o ensino secundário. \\
\hline \multicolumn{3}{|c|}{ Software } \\
\hline $\begin{array}{l}\text { - Universidade de Northern } \\
\text { Illinois }\end{array}$ & http://hackberry.chem.niu.edu/ChemistrySoftware.html & $\begin{array}{l}\text { Lista de software de Química existente na Internet } \\
\text { (algum livre). }\end{array}$ \\
\hline - Imperial College, Londres & http://www.ch.ic.ac.uk/progs.html & Software de Química, desenho e Internet (acesso livre). \\
\hline \multicolumn{3}{|c|}{ Publicações Electrónicas } \\
\hline - Network Science & http://www.awod.com/netsci & \\
\hline - Bull.Chem.Soc.Japan & http://www.syp.toppan.co.jp:8082/Bcsjstart.html & \\
\hline - Journal of Biol. Chemistry & http://www-jbc.stanford.edu/jbc/ & \\
\hline - Electronic Protein Science & http://www.prosci.uci.edu/ & \\
\hline - Chem. Physics Preprints & http://www.chem.brown.edu/chem-ph.html & \\
\hline - Tetrahedron & http://www.elsevier.nl/locate/tis & \\
\hline - Trends Anal. Chem: & http://www.elsevier.nl/freeinfo/trac/Menu.html & \\
\hline - I. Molecular Modelling* & http://derioc1 .organik.uni- & \\
\hline • J. Comp. Aided Mol. Design* & $\begin{array}{l}\text { erlangen.de/info//MOLMOD/jmolinfo.html } \\
\text { http://wucmd.wustl.edu/jcamd/jcamd.html }\end{array}$ & \\
\hline - Betacyte list of e-journals & http://www.betacyte.pair.com/journals.html & Lista de revistas presentes na $W W W$ \\
\hline
\end{tabular}

\section{Conferências Electrónicas}

- Computational Chemistry http://hackberry.chem.niu.edu/ECCC2/

(ECCC- 2)

- Organic Chemistry (ECTOC-1) http://www.ch.ic.ac.uk/ectoc

- Heterocyclic Chemistry (ECHET 96)

- NMR '95 Poster Session http://bellatrix.pcl.ox.ac.uk/nmr/poster.html

- Glycoscience (EGC-1) http://bellatrix.pcl.ox.ac.uk/people/barry_spring95/ barry.gig_econf.html

\begin{tabular}{lll}
\hline & \multicolumn{2}{c}{ Outros } \\
\hline - W3 Search Engines & http://cuiwww.unige.ch/meta-index.html & Índices globais da Internet. \\
- WebElements & http://www.shef.ac.uk:80/ chem/web-elements/ & Tabela periódica interactiva on-line. \\
- ChemExper & http://www.chemexper.be/0 & Catálogos on-line incluindo informação sobre segurança. \\
- Catálogos Fisher Scientific & http://www.fisher1.com/ & \\
\hline
\end{tabular}

-Acesso restrito 
acedam muitos utilizadores. Estão assim em experimentação vários mecanismos de publicação electrónica desde fóruns de discussão livres a sistemas de selecção que envolvem a tradicional revisão anónima e "refereeing".

\section{Revistas electrónicas}

A generalização da Internet não significa que as publicações tradicionais, em papel, vão acabar. O papel é um suporte que apresenta (ainda ?) sólidas vantagens [12]: é mais portátil, permite uma impressão com mais alta resolução do que um monitor electrónico, é mais fácil de percorrer rapidamente e, ainda durante alguns anos, vai ser de acesso mais generalizado e de maior facilidade de integração em bibliotecas de muitos utentes. Por outro lado, a publicação electrónica é muitíssimo mais rápida, tem custos mais reduzidos, permite fazer pesquisas sofisticadas, para além de poder utilizar recursos que não cabem nas dimensões da "página". Em termos de durabilidade, a informação electrónica é praticamente eterna, desde que se criem processos para converter os formatos actuais naqueles que o futuro vier a utilizar. Mas, em termos de médio prazo, não parece ainda possível garantir que a URL de um documento se mantenha inalterada daqui a 5 ou a 10 anos. Por outro lado, é preciso ainda definir em que casos é que uma publicação poderá ser actualizada e em que casos deve permanecer inalterada independentemente dos resultados posteriormente obtidos.

A American Chemical Society (ACS) optou por manter as suas publicações convencionais, complementando-as com material suplementar (agora chamado "de suporte") disponível na Internet aos assinantes. Isto permite-lhe evitar os custos de impressão associados a imagens coloridas, dados espectroscópicos ou descrições experimentais volumosas, sem deixar de disponibilizar essa informação. A Royal Soci- ety of Chemistry tem um projecto de colocar na Internet até final de 1996 os resumos gráficos do Journal of Chemical Society: Chemical Communications.

Outras publicações, como o Bulletin of the Chemical Society of Japan, o Journal of Biological Chemistry ou o Journal of Computer Aided Molecular Design mantêm versões electrónicas integrais em paralelo com as versões tradicionais. Por seu lado, as publicações da família Tetrahedron têm acessíveis a partir de Janeiro de 1996 os resumos gráficos e os índices dos últimos números e, num prazo de dois anos, estarão disponíveis (aos assinantes) os textos integrais.

Mas casos há de revistas exclusivamente electrónicas. O Journal of Molecular Modelling, por exemplo, foi lançado em 1995 e tem acessível, livremente, todos os resumos, enquanto que os conteúdos integrais são facultados apenas mediante assinatura.

\section{Conferências electrónicas}

Nos últimos dois anos tem vindo a ganhar forma um modelo de conferência electrónica na WWW em que as comunicações apresentadas são integralmente públicas e as discussões feitas por correio electrónico ou por um formulário interactivo (ver ECTOC-1 ou ECCC-2, Tabela I). Cada artigo ou poster pode estar localizado no computador pessoal do autor, o que significa que não é requerida proximidade espacial nem entre os participantes nem entre os conteúdos.

Esta modalidade permite apresentar publicamente e submeter a discussão resultados recentes, ainda não publicados num artigo convencional. Comparativamente com os congressos tradicionais, permite uma análise muito mais aprofundada das comunicações, não tem as limitações impostas pela ocorrência simultânea de sessões e é muito menos exigente em termos de custos e de tempo, tanto para a organização como para os participantes. Conferências deste

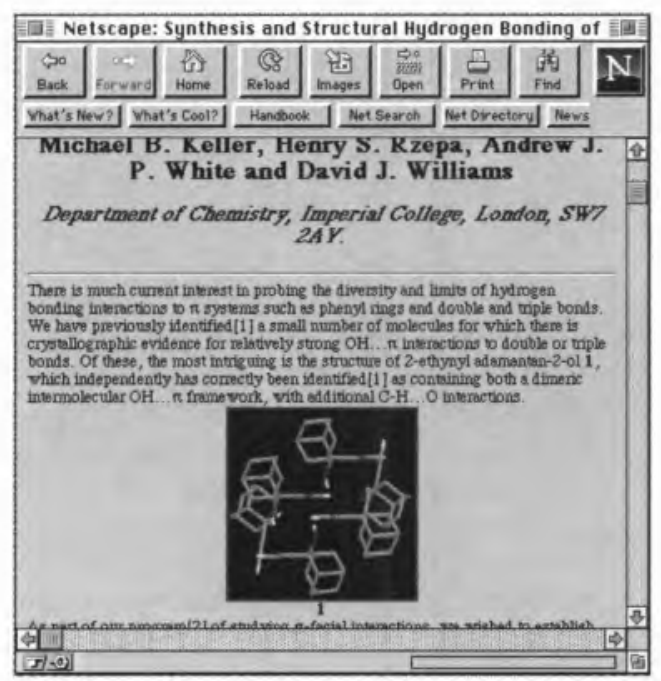

Fig. 1 - Janela do programa Netscape após chamar um poster electrónico apresentado na ECTOC-1 (Junho de 1995)

tipo vão, sem dúvida, generalizar-se e tornar-se em acontecimentos de grande impacto e audiência, passando os congressos tradicionais a valorizar sobretudo o que se relacione com o desempenho e empatia pessoais.

\section{Pesquisas bibliográficas on-line}

A Telnet tem sido muito utilizada para colocar disponíveis bases de dados. É o caso do serviço BIDS (ver Tabela I) que disponibiliza gratuitamente a qualquer utilizador a pesquisa nas tabelas de conteúdos de mais de 15000 publicações científicas a partir do ano de 1981. Para além disso, é possível encomendar a versão integral de qualquer dos artigos cobertos, sendo esta enviada por fax num prazo máximo de 24 horas (desde que se enviem as informações dum cartão de crédito no qual é debitado o custo). Bases de dados comerciais como Chemical Abstracts ou Beilstein estão disponíveis mas o seu acesso não é obviamente gratuito.

\section{Material de ensino on-line}

Imagine-se a quantidade de material de ensino que existe espalhado pelos milhares de Departamentos de Química e Escolas do mundo intei- 
ro... Material acumulado ao longo dos anos para uso interno e que os autores nunca imaginaram publicar por impossibilidade financeira, pelo esforço requerido, pela sua pequena dimensão ou simplesmente porque não... Imagine-se o que seria tornar acessível todo esse material simplesmente ligando à Internet os computadores onde ele se encontra e efectuando algumas simples alterações de formato...! A situação actual não é bem essa (sobretudo por razões de barreira psicológica) mas importa alertar alunos e professores para a impressionante quantidade de material de ensino que já está disponível, tanto ao nível universitário como secundário (ver Tabela I) e que incorpora normalmente as possibilidades "multimédia" do suporte Internet.

Finalmente, convidamos os leitores a experimentar a versão WWW deste artigo localizada na URL http://www.dq.fct.unl.pt/textos/quiminet.html

\section{* Bolseiro de Doutoramento da JNICT Departamento de Química da Faculdade de Ciências e Tecnologia Universidade Nova de Lisboa E-mail:jas@mail.fct.unl.pt}

\section{BIBLIOGRAFIA}

1. B. Sterling, "Short History of the Internet", The Magazine of Fantasy and Science Fiction, Fevereiro de 1993. Ver tam. bém gopher://gopher.isoc.org:70/00/internet/history/ short.history.of.internet

2. E. Krol, "What is the Internet ?", Maio de 1993 ftp://nic.merit.edu/documents/fyi/fyi20.html

3. Amdahl's WWW Hot Topic: Internet 25th Anniversary, 1994, http://www.amdahl.com/internet/events/inet25.html

4. R. Zakon, Hobbes' Internet Timeline v2.2, Agosto de 1995, http://info.isoc.org/guest/zakon/Internet/History/ HIT.html

5. Fonte: Matrix Information and Directory Services, Inc
(MIDS), Fevereiro de 1996, http://www1 , mids.org/ids3/ pr9510.html

6. A.M. Rutkowski, "Bottom-Up Information Infrastructure and the Internet", Founders Day Symposium, University of Pittsburgh, Fevereiro de 1995, http://info.isoc.org:80/speeches/upitt-foundersday.html

7. H.Hahn, R.Stout, "The Internet Complete Reference", Osborne McGraw-Hill, Berkeley, 1994.

8 R. Stembridge, "Chemical Information Resources on the World Wide Web", 210th. Meeting of the American Chemical Society, Chicago, Agosto de 1995. Ver também http:/hackberry.chem.niu.edu:80/Infobahn/Paper23/

9. H. Rzepa, B. Whitaker, M. Winter, I. Chem. Soc, Chem Commun. (1994) 1907. Ver também http:/www.ch.ic.ac.uk/ rzepa/RSC/CC/4_02963A.html

10. M. Keller, H. Rzepa, A. White, and D. Williams, Elec Conf. Tr. Org.Chem., 1995, http://www.ch.ic.ac.uk/ectoc papers $/ 50 /$

11. H. Rzepa, Trends in Analytical Chemistry - Internet Column (Julho de 1995). Ver também http://www.ch.ic.ac.uk /rzepa/TrAC/e-journal.html

12. D. Martinsen, L. Garson, J. Spring, S. Barclay, A. Durniak, and M. Chee, "Using Internet Services to Augment Printed Publications", 210th. Meeting of the American Chemical Society, Chicago, Agosto de 1995. Ver também http:/ pubs.acs.org/presentation/acs9508/

\section{Novos Analisadores de Humidade \\ Modelos HR73 e HG 53 \\ da nossa Representada "METTLER TOLEDO AG"}

Os novos analisadores com lâmpada de halogéneo permitem uma secagem rápida, suave, precisa e controlada da amostra. Tudo o que se evapora sob a lâmpada de halogeneo é registado e armazenado.

Para mais informações, consulte a nossa Direcção Comercial.

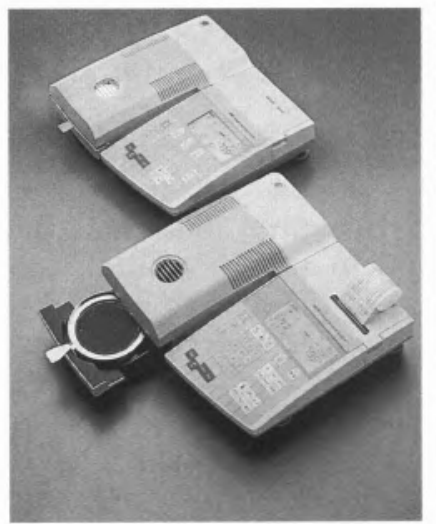




\section{Como ligar à Internet}

Em primeiro lugar precisa de um computador! Nada de especial... É só aconselhável que tenha pelo menos 8 MB de RAM... Macintosh ou PC... tanto faz.

Depois, se o computador está localizado numa Universidade ou num edifício cuja rede interna está ligada à Internet, basta ligar com um cabo (e eventualmente uma placa) o computador à tomada respectiva e é preciso que lhe atribuam um "IP Address".

Mas se pretende ligar o computador a partir de casa ou de outro local qualquer, então precisa de três coisas: uma linha telefónica vulgar, um modem e a subscrição de um serviço comercial de acesso à Internet. Sobre a linha telefónica não há nada a dizer... é a sua linha telefónica habitual, que pode continuar a ser usada para chamadas de voz. Quanto ao modem, que é o aparelho que permite ao computador transmitir e receber dados através da linha telefónica, convém que comunique a uma velocidade de pelo menos 14400 bps e pode adquirilo numa loja de material informático por um preço a partir de 20000\$00. O modem, para além da ligação à Internet ou a outro computador qualquer, possibilita também o envio e recepção de faxes. Quanto ao acesso à Internet, existem empresas que, por uma mensalidade entre $2500 \$ 00$ e 5000\$00, permitem a ligação (SLIP/PPP) esporádica, através de uma chamada telefónica, com direito a 15-20 horas de utilização por mês. De entre as muitas que estão a aparecer destacam-se a Telepac (Tel: 01 - 7907070), PUUG (Tel: 01- 29428 44) e IP (Tel: 01 - 3160328 ).

Para além disto precisa de software. É normalmente software livre, que não precisa de pagar. As empresas que dão acesso fornecem aos seus clientes o software mínimo e instruções sobre como configurá-lo. Se não é esse o seu caso, pode pedir no Serviço de Informática da sua Universidade ou a alguém que já esteja ligado. E quando já tiver algum software para que o sistema funcione, pode adquirir o resto através da Internet, onde quase tudo o que vai necessitar está disponível (ver Tabela).

Este software pode ser classificado em três tipos: de comunicação, de rede e auxiliares. O software de comunicação é praticamente "invisível" e serve para ligar o seu computador à rede (é até provável que parte dele já exista no seu sistema operativo). O software de rede inclui programas para trabalhar com cada um dos serviços da Internet (E-mail, WWW, FTP, etc) - ver "Química na Internet". Os auxiliares permitem visualizar (ou ouvir) ficheiros cujo formato não é directamente utilizável pelo software de rede; por exemplo, pode importar um ficheiro de som através dum programa de WWW mas para o ouvir precisa de um programa auxiliar.

Na Tabela abaixo estão listados programas para cada uma das funções descritas e o local onde podem ser adquiridos na
Internet.

Só mais uma palavra relativamente aos auxiliares. É muito útil configurar o programa de WWW (Netscape, por exemplo) para que quando importar ficheiros de determinados tipos, seja aberto o programa auxiliar respectivo. Por exemplo, para que quando importar um ficheiro com coordenadas moleculares em formato pdb, seja imediatamente aberto o programa visualizador que permite explorar a estrutura em três dimensões.

Para fazer esta configuração no programa Netscape deve escolher o menu "Options", depois "General Preferences" e depois "Helpers". Deve então introduzir uma nova entrada com "Mime type: chemical, Subtype: $x$-pdb, Extension: pdb". De seguida deve escolher "Browse" e seleccionar o programa auxiliar (RasMac ou RasWin, p.ex.). Recentemente foi lançado em alternativa o "plug-in" Chime que é introduzido na directoria "Plug-ins" do Netscape 2.0 fazendo com que este reconheça e visualize moléculas em formato pdb na sua própria janela. Analogamente, pode configurar para outros tipos de ficheiros.

E pronto. Quando tudo estiver instalado só tem que ligar o modem e usar o programa que pretender: o software de comunicação encarrega-se de estabelecer a ligação. Como vê, é simples... E lembre-se que antes de si já vários milhões de pessoas fizeram o mesmo... 
Tabela - Programas para a Internet e sua localização*

\begin{tabular}{|c|c|c|c|}
\hline Função & Macintosh & Windows 3.1 & Windows 95 \\
\hline Comunicação & $\begin{array}{c}\text { InterSLIP } \\
\text { ftp://ftp.intercon.com//Inter } \\
\text { Con/sales/InterSLIP } \\
\text { MacPPP } \\
\text { ftp://merit.edu/pub/ppp/mac } \\
\text { /macppp2.0.1.hqx }\end{array}$ & $\begin{array}{l}\text { Trumpet Winsock } \\
\mathrm{ftp}: / / \text { ttp.coast.net/SimTel/wi } \\
\text { n3/winsock/twsk20b.zip }\end{array}$ & $\begin{array}{l}\text { Trumpet Winsock for Win95 } \\
\mathrm{ftp}: / / \text { ttp.enterprise.net/pub } \\
\text { /mirror/winsock-1/Windows95 } \\
\text { WinSock/twsk95b1.zip }\end{array}$ \\
\hline WWW & \multicolumn{3}{|c|}{ Netscape Navigator http://home.netscape.com/comprod/mirror/client_download.html } \\
\hline E-mail & $\begin{array}{l}\text { Eudora } \\
\text { ftp://ftp.qualcomm.com/que } \\
\text { st/mac/eudora/1.5/ }\end{array}$ & $\begin{array}{c}\text { Eudora } \\
\text { ftp://ftp.ua.pt/pub/winsock/ } \\
\text { mail/eudor152.zip }\end{array}$ & $\begin{array}{c}\text { e-Mail Notify } \\
\text { for Windows } 95 \\
\mathrm{ftp}: / / \text { papa.indstate.edu//win } \\
\text { sock-I/Windows95/Mail/ } \\
\text { e111 w95.zip }\end{array}$ \\
\hline Telnet & $\begin{array}{c}\text { NCSA Telnet } \\
\text { ftp://ftp.ncsa.uiuc.edu/Mac/ } \\
\text { Telnet/Telnet2.6/ }\end{array}$ & $\begin{array}{l}\text { Ewan } \\
\text { ftp://ftp.funet.fi//pub/mirrors } \\
\text { /ftp.winsite.com/pub/pc/win } \\
\text { 3/winsock/ewan105.zip }\end{array}$ & $\begin{array}{c}\text { QVT Winsock 32-bit } \\
\text { http://download.netex.net/ } \\
\text { w95/windows95/internet/qv } \\
\text { tn3989.zip }\end{array}$ \\
\hline FTP & $\begin{array}{c}\text { Fetch } \\
\text { ftp://ftp.the.net/mirrors/ftp.u } \\
\text { texas.edu/tcpip/fetch-30-fat.hqx }\end{array}$ & $\begin{array}{c}\text { WS-FTP 16-bit } \\
\text { ftp://papa.indstate.edu/wins } \\
\text { ock-l/ftp/ws_ftp.zip }\end{array}$ & $\begin{array}{c}\text { WS-FTP 32-bit } \\
\text { ftp://ftp.csra.net/pub/win32/ } \\
\text { ws_ftp32.zip }\end{array}$ \\
\hline Archie & $\begin{array}{c}\text { Anarchie } \\
\text { ftp://hyperarchive.Ics.mit.ed } \\
\text { u/info-mac/comm/tcp/ } \\
\text { anarchie-16.hqx }\end{array}$ & $\begin{array}{c}\text { WS-Archie } \\
\text { ftp://ftp.coast.net/SimTel/wi } \\
\text { n3/winsock/wsarch08.zip }\end{array}$ & $\begin{array}{c}\text { W95 Comand Line Archie } \\
\text { http://download.netex.net/ } \\
\text { w95/windows95/internet/w9 } \\
\text { 5_archie.zip }\end{array}$ \\
\hline Usenet & $\begin{array}{c}\text { Internews } \\
\mathrm{ftp}: / / \mathrm{ftp} . c 0 m m e r c e . c o m / \mathrm{pub} \\
\text { /mac/internews-1.0.7.hqx }\end{array}$ & $\begin{array}{c}\text { Free Agent } \\
\text { ftp://ftp.ua.pt/pub/winsock/ } \\
\text { news/fagent10.zip }\end{array}$ & $\begin{array}{c}\text { News for Windows } 95 \\
\text { ftp://ftp.enterprise.net/pub/ } \\
\text { mirror/winsock-1/Windows95 } \\
\text { /News/news073.zip }\end{array}$ \\
\hline Expandir ficheiros & $\begin{array}{c}\text { Stuffit Expander } \\
\text { ftp://hyperarchive.Ics.mit.ed } \\
\text { u/info-mac/cmp/stuffit-expander-352.bin }\end{array}$ & $\begin{array}{c}\text { winzip } \\
\text { http://www.ch.ic.ac.uk/prog } \\
\text { s.html }\end{array}$ & \\
\hline Som & $\begin{array}{c}\text { Soundmachine } \\
\text { http://www.ch.ic.ac.uk/prog } \\
\text { s.html }\end{array}$ & $\begin{array}{l}\text { Wham Sound Program } \\
\text { http://www.ch.ic.ac.uk/pro } \\
\text { gs.html }\end{array}$ & \\
\hline Estruturas molec. 3D & $\begin{array}{c}\text { RasMac } \\
\text { http://www.ch.ic.ac.uk/progs.html }\end{array}$ & $\begin{array}{c}\text { RasWin } \\
\text { http://www.ch.ic.ac.uk/progs.html }\end{array}$ & \\
\hline \multicolumn{4}{|c|}{ Chemspace Chime http://www.mdli.com/chemscape/chime/chime.html } \\
\hline Esquemas reaccionais & $\begin{array}{c}\text { ISIS/Draw } \\
\text { http://www.ch.ic.ac.uk/progs.html }\end{array}$ & $\begin{array}{c}\text { ISIS/Draw } \\
\text { http://www.ch.ic.ac.uk/progs.html }\end{array}$ & \\
\hline Vídeo QuickTime & $\begin{array}{c}\text { QuickTime } \\
\text { http://www.ch.ic.ac.uk/progs.html }\end{array}$ & $\begin{array}{c}\text { QuickTime } \\
\text { http://www.ch.ic.ac.uk/progs.html }\end{array}$ & \\
\hline Imagens & $\begin{array}{c}\text { GraphicConverter } \\
\text { http://www.ch.ic.ac.uk/progs.html }\end{array}$ & $\begin{array}{c}\text { LView Pro } \\
\text { http://www.ch.ic.ac.uk/progs.html }\end{array}$ & \\
\hline
\end{tabular}

·Todas as localizaçôes da tabela foram verificadas após 20.02.96. É provável que rapidamente se desactualizem. 
